\title{
SIR GAWAYN AND PE GRENE KNY3T: POLÍTICA DE LA FICCIONALIZACIÓN EN LA INGLATERRA EXPANSIONISTA MEDIEVAL
}

\author{
Carina Menán y Cecilia Lasa* \\ Universidad de Buenos Aires (Argentina)
}

El romance medieval Sir Gawayn and pe Grene Knyzt emerge en el contexto de la colonización de Gales por parte de Inglaterra. El poema inglés se construye a partir de una serie de oposiciones en las que la cultura en expansión se identifica con el mundo de lo civilizado en contraste con la barbarie representada por el caballero verde y los espacios que este habita. Sin embargo, el poema se reviste de un carácter paródico que expone las contradicciones de las políticas ideológicas de extensión territorial al poner en cuestión el binarismo construido por el mismo romance. La dimensión desestabilizadora de la parodia, no obstante, es cancelada por otro procedimiento formal: la caracterización de Gawayn como el caballero inglés que encarna los valores de la civilización expansionista.

Palabras claves: Inglaterra medieval - Expansionismo - Gales - Política - Parodia

SIR GAWAYN AND PE GRENE KNY3T: POLITICS OF FICTIONALIZATION IN MEDIEVAL EXPANSIONIST ENGLAND

The Medieval romance Sir Gawayn and pe Grene Knyzt emerges in the context the English conquest of Wales. The English poem is constructed through a series of oppositions in which the expanding culture identifies with the civilized world, which stands in contrast to the barbarity embodied in the Green Knight and the spaces he inhabits. Nevertheless, the poem shows a parodic feature that exposes the contradictions in the policies of territorial expansion by questioning the binarism constructed by the romance itself. The destabilizing dimension of the parody, however, is cancelled by another formal resource: Gawayn's characterization as an English knight that personifies the values of the expansionist civilization.

Keywords: Medieval England - Colonization - Wales - Parody - Romance

Artículo Recibido: 23 de Noviembre de 2017

Artículo Aceptado: 15 de Marzo de 2018

\footnotetext{
*E-mail: cmenan@hotmail.com; cecilia_ev_lasa@hotmail.com
} 


\section{Introducción}

1 romance medieval Sir Gawayn and pe Grene Kny3t (en adelante, SGGK) surge en el
contexto de la colonización de Gales por parte de Inglaterra a fines del siglo XIV. Sus
versos tienen como objeto el viaje de Gawayn, sobrino del rey Arthure y también caballero de su corte, a tierras desconocidas que remiten al suelo galés como parte de un reto desencadenado por una criatura verde: este ser extraño desafía a los vasallos artúricos a decapitarlo para luego de un año reencontrarse con él si logra sobrevivir, como efectivamente sucede. En su intervención en el escenario expansionista, esta pieza ficcional se ideologiza al presentar un conjunto de oposiciones en el que la cultura en expansión se identifica con el mundo de lo civilizado en contraste con la barbarie representada por el caballero verde y los espacios a los que este pertenece. Así se figura la conquista de Gales: su salvajismo encarna una amenaza que la civilización inglesa debe domesticar. Sin embargo, el poema se reviste de un carácter paródico que expone las contradicciones de las políticas ideológicas de extensión territorial al poner en cuestión el binarismo construido por el mismo romance. La dimensión desestabilizadora de la parodia, no obstante, es neutralizada por otro procedimiento formal que la absorbe: la caracterización de Gawayn como el caballero que encarna los valores de la 
Inglaterra expansionista. Mientras que su pasaje por el mundo galés le permite ver que la otredad cifrada en el romance es una operación política e ideológica del ímpetu colonialista, su reincorporación a la corte al final del poema implica un desenlace conservador necesario para fortalecer la monarquía inglesa que, por medio de la ampliación de territorio, intenta materializar su supremacía. El reconocimiento de la debilidad propia por parte de Gawayn-que la corte ignora- evidencia un texto que no puede borrar totalmente las fisuras de su propio relato aunque es incapaz de proponer una alternativa formal y política a la autocrítica de la parodia dado que él mismo se ha tornado mecanismo colonizador.

Para el estudio de la hipótesis presentada, dividiremos la exposición en tres ejes de análisis: en primer lugar, exponemos una sucinta reseña histórica sobre el contexto de producción del romance, noción que luego, en un segundo momento, conceptualizamos a la luz de los acontecimientos socioculturales referidos; en tercer lugar, estudiamos cómo se entrelazan cuestiones políticas con operaciones formales sobre el género. Esta organización da cuenta de la perspectiva metodológica desde la cual conducimos nuestro análisis: nuestra lectura del romance intenta no reducirlo a un ejemplar de procesos históricos mayores o a un catálogo de recursos retóricos abstraído de las condiciones socioeconómicas, políticas y culturales que lo originan. SGGK, como producción textual de carácter ficcional, no es ajeno al momento histórico en el que se inserta y al que se dirige, vínculo que se visibiliza no solo en su contenido, sino en su forma. Tratamos de leer, como sintetiza Funes, «la incidencia de la historia en lo específico del arte verbal» ${ }^{1}$ : en nuestro abordaje de este romance medieval, elegimos «no la articulación de efectos de sentido distribuidos sobre la superficie de los textos sino adentrar[nos] en los textos, ahondar en ellos, no en busca de profundidad metafísica sino de su espesor histórico» ${ }^{2}$. El proceso de ficcionalización de los insumos textuales cobra una forma particular que, en su relación con el marco histórico en el que se gesta, condensa posicionamientos ideológicos. Esta dinámica, que constituye también nuestro objeto de escrutinio, se nutre de aquello sobre lo que versa el texto su contenido- como del modo en que lo hace -su forma-. Por eso, el hecho de que SGGKcanalice la voz de espacios y personajes que la Inglaterra del siglo XIV considera periféricos -tales como el territorio galés o una mujer- no lo dota, necesariamente, con un tono contestatario: su presencia en el texto cobra sentido de acuerdo con la manera en que participa del conflicto ficcionalizado, que no escapa a las coordenadas históricas en las que se desarrolla.

\footnotetext{
${ }^{1}$ Funes, Leonardo, «Medievalismo en el otoño de la Edad Teórica. Consideraciones parciales sobre la operación filológica», en Perspectivas actuales de la investigación literaria, Editorial de la Facultad de Filosofía y Letras de la Universidad de Buenos Aires, Buenos Aires, 2011, p. 70 (pp. 45-72).

${ }^{2}$ Ibidem, p. 61.
} 


\section{Contexto histórico}

La fecha de composición de $S G G K$-generalmente localizada al último cuarto del siglo XIVcoincide con violentos intentos ingleses de afianzar la conquista de Gales. De acuerdo con Arner, este proyecto, que se alinea con una política expansiva de distintos modos y grados de injerencia a lo largo de varios siglos, se enfrenta, no obstante, con activa resistencia ${ }^{3}$. En virtud de dicho contexto de producción, la crítica concibe el poema como un instrumento cuya ideología "attempted to reformulate readers' conceptions of themselves and of their neighbors and thus shape their perceptions of how to negociate English Welsh conflicts» ${ }^{4}$. El texto, desde una perspectiva centrada en la cultura dominante, participa en la edificación de un clima político favorable a la expansión y la conquista por parte de los ingleses sobre el territorio galés. El momento en el que el romance emerge es, en realidad, un punto climático en el proceso colonizador.

Hasta comienzos del siglo XIII, el estatuto de Gales con respecto a Inglaterra es periférico. La región para los conquistadores no constituye más que, en palabras de Davies, «a series of well behaved and amenable protectorates, satellites, and colonial annexes around the periphery of the English kingdom ${ }^{5}$. Este reino comienza a tomar forma ya en 1066, en el contexto de la invasión a partir de la que el duque de Normandía Guillermo II adopta la investidura de soberano como Guillermo I de Inglaterra. Dada la naturaleza anglonormanda de esta corte, sus intereses y proyecciones se concentran en los suelos inglés y francés. Davies señala que, para esta monarquia, Gales es tan solo parte de «the outlying and not hugely rewarding parts of the British Isles» ${ }^{6}$. El historiador agrega que recién entre los reinados de Enrique II y Eduardo I se cambia el esquema de conquista y las campañas se presentan como el mecanismo para consolidar control de los márgenes ${ }^{7}$. Son diversos los motivos por los que se produce esta modificación en la percepción acerca de las formas de ejercer dominación. En 1204 la monarquía inglesa pierde

\footnotetext{
3 Arner, Lynn, «The Ends of Enchantment: Colonialism and Sir Gawain and the Green Knight», Texas Studies in Literature and Language. Vol. 48, No. 2, verano 2006 (pp. 79-101), p. 79.

4 «intentaba reformular las concepciones de los lectores acerca de sí mismos y de sus vecinos y así influir sus percepciones sobre cómo negociar los conflictos entre ingleses y galeses». Ibidem, p. 80. Esta y todas las traducciones de textos críticos en inglés nos pertenecen.

5 «una serie de protectorados, satélites y anexos coloniales rodeando la periferia del reino inglés, dóciles y bien dispuestos». Davies, Robert, The First English Empire. Power and Identity in the British Isles 1090. The Ford Lectures Delivered in the University of Oxford in Hilary Term 1998, Oxford University Press, Oxford, New York, 2000, p. 192. 6 "áreas periféricas y no demasiado ventajosas de las islas británicas». Davies, R.R., Domination and Conquest: The Experience of Ireland, Scotland and Wales, 1100-1300, Cambridge University Press, Cambridge, 1990, p. 111.

7 Ibidem, p. 112.
} 
sus tierras en el norte de Francia, lo que la conduce a prestar más atención a su dominio sobre las islas británicas. Simultáneamente, se organizan en Gales tentativas de unificación por parte de los reinos que la componen -entre ellos, Gwynedd, uno de los más rebeldes, ubicado al noroeste-. Estas resistencias alteran el equilibrio de poder y la relación con la aristocracia inglesa allí establecida y con la autoridad regia. Por otro lado, la administración anglonormanda, que vuelca ahora sus intereses hacia el interior del territorio de las islas, adquiere una mayor complejidad y un desarrollo burocrático que le otorga la posibilidad de expansión ${ }^{8}$.

De la mano del refuerzo de las acciones militares, políticas y administrativas ejecutadas para concretar la conquista, se afianza una «fractura cultural» $\mathrm{O}$ «the distinction between what contemporaries occasionally called a regio composita and a regio barbara» ${ }^{9}$. Esta división es constitutiva de la construcción de identidades que se consolidan en este período, tales como «Inglaterra»y «los ingleses» ${ }^{10}$. La cultura hegemónica es la que transmite su perspectiva sobre otras identidades, como la galesa, y esta construcción repercute directamente en lo político:

But for the most part they [Irish \& Welsh's political leaders] were regarded as culturally too backward and politically too immature to be admitted on a regular basis into the ambit of an England-centred political culture. Instead they cultivated their own political mythologies and aspirations and could deploy them on occasion to promote their sense of oppression, disinheritance, and exclusion ${ }^{11}$.

La tajante distinción de sí con respecto a los otros es el pilar para formaciones identitarias caracterizadas por relaciones jerárquicas de dominación, tal como la que se observa entre Inglaterra-que se identifica con el «centro»-y Gales como su periferia. El poema medieval SGGK participa activamente en la consolidación de estas construcciones, no solo por las particularidades de la trama y de los aspectos formales que conforman a este texto, sino también por las características genéricas que lo constituyen en romance.

\footnotetext{
${ }^{8}$ Ibidem, p. 113.

9 «la distinción entre lo que los contemporáneos ocasionalmente llamaban una regio composita y una regio barbara». Davies, Robert, op. cit., p. 119.

${ }^{10}$ Ibidem, p. 192.

11 «Pero en su mayoría [a los líderes políticos irlandeses y galeses] se los veía como demasiado retrasados en términos culturales e inmaduros en exceso en lo que respecta a la política como para admitirlos de manera regular en el ámbito de una cultura política centrada en Inglaterra. En su lugar, cultivaban sus propias mitologías y aspiraciones políticas y podían implementarlas ocasionalmente para promover su sentido de opresión, desarraigo y exclusión». Ibidem, p. 194.
} 


\section{El romance medieval y el personaje de Gawayn}

El romance medieval ocupa un lugar de relevancia en la historia literaria, ya sea por la popularidad y variedad que lo caracteriza en el contexto en el que surge, como por sus derivaciones en los períodos históricos subsiguientes. Desde esta perspectiva, Jameson traza su desarrollo desde su génesis en la chanson de geste hasta, por ejemplo, el western americano. El crítico registra los comienzos del romance en el siglo XII, cuando la nobleza feudal comienza a concebirse a sí misma como una clase universal:

Romance may then be understood as[...Ja symbolic answer to the question of how my enemy can be thought of as being evil, that is as other than myself and marked by some absolute difference, when what is responsible for his being so characterized is simply the identity of his own conduct with mine, which challenges, points of honor, tests of strength - he reflects as in a mirror image ${ }^{12}$.

El romance, entonces, es expresión de los valores identitarios de la clase feudal dominante. Como observa el crítico, la universalidad de la autopercepción de esta clase está acompañada de una valoración positiva de lo propio y una apreciación negativa de lo ajeno, que encarnan el bien y el mal, respectivamente. Esta lectura sociológica imprime una carga ideológica sobre constantes temáticas del género tales como «the knight errant, the beautiful endangered lady, the lost heir, the trial of prowess or virtue, the pact gone wrong, the monstruous, the magical, and/or disguised challenger, the journey to the otherworld, the joyous return of the prodigal and the reintegrative celebration» ${ }^{13}$. Estos rasgos se encuentran atravesados por el posicionamiento político de una clase social que, en el caso de las islas británicas, tiene su centro en la corte anglonormanda que consolida su identidad, se fortalece y expande en los siglos XII, XIII y XIV.

En lo que respecta a la tradición del romance en el territorio insular, el género ingresa de la mano de la conquista normanda de 1066. Dado que el lenguaje de la corte es en principio anglonormando - una variedad antigua del francés-o bien latín, los primeros textos se escriben

\footnotetext{
${ }^{12}$ «Al romance, entonces, se lo puede entender como [...] una respuesta simbólica a la pregunta de qué modo se concibe a mi enemigo como un ser perverso, es decir, como un otro distinto de mí y marcado por alguna diferencia absoluta, cuando en realidad lo que es responsable de que sea caracterizado así es simplemente la identidad de su propia conducta con la mía, a la que - desafíos, cuestiones de honor, pruebas de fuerza - él refleja como en una imagen especular». Jameson, Fredric, «Magical Narratives: Romance as Genre», en New Literary History, Vol. 7, No. 1, Critical Challenges: The Bellagio Symposium, Otoño, 1975 (pp.135-163), p. 161.

13 «el caballero errante, la hermosa damisela en apuros, el heredero perdido, la prueba de coraje o de virtud, el pacto que sale mal, lo monstruoso, el adversario mágico y/o encubierto, el viaje al más allá, el feliz retorno del pródigo y la celebración reparadora», Chism, Christine, «Romance», en Scanlon, Larry (ed.), The Cambridge Companion to Medieval English Literature 1100-1500, Cambridge University Press, Nueva York y Cambridge, 2009, (pp. 57-69), p. 57.
} 
en estas lenguas; la escritura del romance en dialectos del inglés medio se da más bien tardiamente ${ }^{14}$. Son los escritores que emplean estos dialectos quienes «seized upon, redirected, parodied, and criticized the conventions of their sources in an astonishing variety of registers, from the blunt utility of King Horn to the intricacy of Sir Gawain and the Green Knight ${ }^{15}$. En este último, por ejemplo, tanto el caballero verde -cuya identidad se corresponde con la de Lord Bertilak, quien brinda hospedaje al joven de la corte artúrica en las inhóspitas tierras que visitacomo la señora Bertilak se encargan de burlar a la corte y a Gawayn, respectivamente, por no estar a la altura de la reputación que romances previos les han endilgado.

Esta apropiación, sin embargo, no es la única que se produce en $S G G K$ : la figura de Gawayn pertenece a una antigua tradición oral celta al igual que la del rey Arthure y el resto de su corte, aunque la cultura dominante contemporánea a este texto se la atribuye como elemento integrante de su tradición propia ${ }^{16}$. Tal estrategia está visiblemente ligada a una conquista que busca la dominación de los márgenes en el marco de un proyecto en el que se cruzan variables políticas, económicas y culturales. El pasaje de Gawayn de una identidad celta a otra anglonormanda se produce luego de atravesar otros espacios geográficos y genéricos: a principios del siglo XII, es una figura cuya fama se ha extendido a la cultura eclesiástica y letrada europea, pero su popularidad como héroe se ocasiona en la numerosa afluencia a las islas de romances en francés y anglonormando, que se extiende hasta principios del XIII. Posteriormente, durante el siglo XIII, surgen y circulan en Inglaterra una docena de romances en inglés medio que Gawayn protagoniza como el caballero principal de la corte del rey Arthure. Los romances así lo describen: «the Arthurian knight most dedicated to masculine adventure and competition» ${ }^{17}$, «a distinctive model of masculinity [...] a magnet for the desire of a long sequence of women» ${ }^{18}$, o "the unblemished paragon of chivalric virtue» ${ }^{19}$. Es su versatilidad, entonces, el rasgo que lo define como personaje y son estas diversas características las que establecen un diálogo, muchas veces paródico, con el protagonista de $S G G K$.

\footnotetext{
${ }^{14} \mathrm{Ibidem}$, p. 58.

15 «aprovecharon, redirigieron, parodiaron y criticaron las convenciones de sus fuentes en una sorprendente variedad de registros, desde la franca utilidad de King Horn a la complejidad de Sir Gawain and the Green Knight». Ibidem, p. 59.

${ }^{16}$ Arner, Lynn, op. cit. p. 83; Davies. R. R., op. cit. p.2; Hahn, Thomas, "Gawain and popular chivalric romance in Britain», en Krueger, Roberta L. (ed.), The Cambridge Companion to Medieval Romance, Cambridge University Press, Cambridge, 2004, p. 218 (pp. 218-234).

17 «el caballero artúrico más dedicado a la aventura y competencia masculinas». Hahn, Thomas, ibidem, p. 219.

18 «un modelo de masculinidad distintivo [... ] un imán para el deseo de una larga secuencia de mujeres». Ibidem, p. 220.

19 «el modelo inmaculado de virtud caballeresca». Ibidem, p. 221.
} 


\section{Forma y política de la parodia}

Lo paródico en $S G G K$ se inscribe a nivel formal a partir de la caracterización de personajes, la ambientación y el empleo de figuras retóricas para desarticular el romance medieval como género. Según Tynianov, «[1]a esencia de la parodia consiste en la mecanización de un procedimiento determinado» ${ }^{20}$. Un artificio paródico supone, entonces, una conciencia de escritura que retome modos de composición a los cuales coloca como objeto de interpelación. Al hacerlo, debido a la mecanización a la que los somete, demuestra su caducidad. Dicha conciencia está presente en el romance de fines del siglo XIV mediante una voz narrativa que conmina a los receptores del poema a escuchar

this laye [...]

As hit is scad and stoken

In stori stif and strange,

With lel letteres loken,

In londe so has ben lange ${ }^{21}$ (30, 33 36).

Esas letras entrelazan temáticas propias del romance tales como las aventuras de caballería, la presencia de sucesos sobrenaturales y la vida cortesana, de modo tal que la manipulación del género constituye un recurso que comienza a exhibir su obsolescencia ${ }^{22}$. Ya la escena inicial de $S G G K$ expone aquellas características: en el contexto de la celebración de Navidad, abundante en alimentos, dádivas y entretenimientos (60 80), se presenta a un «Arthure [...] / [...] sumquat childgered ${ }^{23}$ ( 85 86), inquieto (87 88), que solo dará inicio al ágape bajo la condición de que se lo entretenga con una justa (91 99) o bien con relatos de aventuras, maravillosos o de

\footnotetext{
${ }^{20}$ Tynianov, luri, «Tesis sobre la parodia», en Volek, Emil (ed. y trad.), Antología del formalismo ruso y el grupo de Bajtin. Polémica, historia y teoría literaria. Vol. I Polémica, historia y teoría literaria, Fundamentos, Madrid, 1992, p. 169 (pp. 169-170).

${ }^{21}$ «mi cuento que en tinta es un relato audaz y potente, mediante palabras que, unidas, duran fuerte y mucho». Esta y todas las citas provienen de la edición de Sir Gawayn and pe Grene Knyzt al cuidado de Simon Armitage. Armitage, Simon (ed.), Sir Gawain and the Green Knight, W. W. Norton \& Company, Londres y Nueva York, 2007. ${ }^{22}$ Tomachevski, Boris, «La construcción de la trama», en Teoría de la literatura, Trad. Suárez, Marcial, Akal, Madrid, 1982, pp. 210-211 (pp. 179-211).

${ }_{23}$ «Arthure, casi infantil». Armitage, Simon (ed.), op. cit.
} 
caballería -nuevamente, el romance se vuelve sobre sí mismo haciendo alusión a su propio propósito-. La caracterización del monarca como un niño inmaduro vehiculiza el primer elemento paródico en tanto el catálogo de sus deseos, típicamente tematizados por el romance, se reduce a una serie de caprichos.

El tono objetante de la voz narrativa se actualiza mediante la aparición del caballero verde luego del pedido infantil del soberano. El recién llegado vocifera los motivos por los que ha irrumpido en la corte:

Bot for the los of the, lede, is lyft up so hyghe, And thy burgh and thy burnes best ar holden, Stifest under stel gere on stedes to ryde,

The wyghtest and the worthyest of the worldes kynde, Preve for to play wyth in other pure laykes, And here is kydde cortaysye, as I haf herd carp, And that has wayned me hider, iwyis, at this tyme ${ }^{24}$ (258 265).

La parodia se dibuja en sus palabras a causa del contraste entre el modo hiperbólico en el que se hace eco de la reputación de los caballeros de la Mesa Redonda y su observación empírica. Los seguidores de Arthure, sospechosamente «al stouned at his steven and stonstil seten / In a swoghe sylence» $^{25}(241 \sim 242)$ ante la llegada del ser sobrenatural, no responden al desafío que este propone: esta figura monstruosa se someterá a una decapitación si y solo si el vasallo del soberano que la ejecute lo busque al pasar un año en el caso de sobrevivir al juego. El intruso se burla frente a la pasividad de los jóvenes que rodean al monarca:

"What, is this Archures hous,» quoth the hathel thenne, "That al the rous rennes of thurgh ryalmes so mony?

Where is now your sourquydrye and your conquestes, Your gryndellayk and your greme and your grete wordes? Now is the revel and the renoun of the Rounde Table Overwalt wyth a worde of on wyyes speche, For al dares for drede wichoute dyne schewed! $\Downarrow^{26}$ (309 312)

\footnotetext{
24 «Pero dado que se te aclama fuerte al unísono, y que a tu castillo y tu hermandad se los llama los mejores, los hombres más fuertes en sus monturas, los caballeros más fuertes del mundo, tanto en la competencia como en el combate verdadero, y dado aquí se defiende, según dicen, la cortesía, me encuentro intrigado, atraído hasta tu puerta en esta oportunidad». Armitage, Simon (ed.), op. cit.

25 "como estatuas en sus asientos, mudos y rígidos». Armitage, Simon (ed.), op. cit.

${ }^{26}$ ««¿Así que esta es la corte de Arthure», se mofó, «cuyas virtudes reverberan a lo largo de vastos reinos?

¿Dónde está la fortaleza y el arrojo por los que son tan famosos?
} 
El texto, a través de las observaciones del personaje verde, parece sugerir que los cantares de gesta y sus relatos - protagonizados por el valor de los caballeros y su lealtad incuestionable al soberano- han quedado atrás y que, pese a su fama, ellos son tan imberbes como el rey. Entonces, se observa cómo el retrato de la corte de Camelot de los versos inaugurales manipula los aspectos prototípicos del romance medieval y los ridiculiza de modo solapado mediante el intruso en el banquete artúrico. Lo sobrenatural, sobre lo que el romance se ha arrogado propiedad, deviene así en el modo en que se expresa la parodia.

Asimismo, lo paródico en $S G G K$ posee alcances políticos. Arner recuerda que el poema se escribe en «a period during which the English were attempting to complete their colonization of Wales, while the Welsh violently opposed such domination» ${ }^{27}$, expresada en asesinatos y sustracción de territorios y recursos de subsistencia 28 . De hecho, el suelo galés es el que habita el caballero verde y hacia el que se dirige Gawayn al año de cumplirse el reto ya que él es quien decapita fútilmente a la figura sobrenatural. Tanto la ambientación como los personajes que lo pueblan son descriptos en términos de lo que Inglaterra considera como incivilizado: un paisaje inhóspito y sus criaturas indómitas se oponen a la protección que brinda la corte (713 733). Lilley explica que ya en el siglo XII cronistas ingleses «saw the Welsh and the Irish as «inferior» peoples living on "peripheral» lands» ${ }^{29}$ y señala, además, cómo la corte anglonormanda construye a través de la voz de estos cronistas su estatus de superioridad sobre la creencia de que "the Welsh and the Irish were not urbanized, [...] they lived without «towns, villages or castles»»30. Esto se condice con el modo en que $S G G K$ construye la extranjeridad de la periferia. Tal como refiere Benson, el caballero verde es en estos márgenes un dispositivo fundamental, ya que «[he] represents the stock enemy of knighthood in medieval romance, the wild man, a figure who opposes knights and who strives against the values represented by courts» ${ }^{31}$. El romance

¿Y la valentía asombrosa y las enormes bocas fanfarronas?

La altísima reputación de la Mesa Redonda

derribada y aplastada por un extraño. ¡Qué escándalo!»»».

Armitage, Simon (ed.), op. cit.

27 «un período en el que los ingleses intentaban completar la colonización de Gales, mientras los galeses se oponían violentamente a tal dominación». Arner, Lynn, op. cit., p. 79.

${ }^{28}$ Ibidem, p. 81.

29 "consideraban a los galeses y a los irlandeses como pueblos «inferiores» en tierras «periféricas»». Lilley, Keith, «Imagined Geographies of the «Celtic Fringe» and the Cultural Construction of the 'Other' in Medieval Wales and Ireland», en Harvey, David, Jones, Rhys, McInroy, Neil y Milligan, Christine. Celtic Geographies. Old Culture, New Times, Routledge, Londres y Nueva York, 2005, p. 23 (pp. 21-36).

30 «los galeses y los irlandeses no estaban urbanizados, [...] no vivían en «aldeas, pueblos o castillos»». Ibidem, p. 25.

31 «representa el enemigo de la caballería en el romance medieval, el hombre salvaje, una figura que se opone a los caballeros y lucha en contra de los valores que las cortes representan». Arner, Lynn, op. cit., p. 88. 
constituye el género en el que se cristaliza al personaje de Gawayn como exponente de los valores de la aristocracia feudal: el protagonista es un modelo de humildad, lealtad y buenos modales, tal como se observa en el pedido de permiso a su soberano para aceptar el desafío del ser sobrenatural (339 353) y marchar, al año, a sus tierras. Asimismo, encarna las virtudes del caballero cristiano - «fraunchyse and felawschyp [...]; [...] clannes and [...] cortaysye, / and pité»32 (652 654)-: en su escudo hay un pentáculo que las simboliza y que además porta la imagen de María (648 649). Haber dado su palabra, por otro lado, lo conducirá a desafiar sus propios temores y debilidades, y a vencer diversas tentaciones. Sin embargo, es justamente la parodia la que desestabiliza esos ideales y, por lo tanto, su contraposición con aquellos representados por el caballero verde. Tal como sintetiza Caramés Lage, «el retador [...] reta no solo al rey, sino a toda la sociedad» ${ }^{33}$. En este sentido, el caballero verde, a partir de su caracterización y de la puesta en jaque de la courtaysye «como representación tangible de ese ideal de armonía al que las cortes y el pensamiento medievales aspiraban» 34 , pone en ridículo y expone la política expansionista inglesa. La parodia se presenta en esta oportunidad como procedimiento para el desmantelamiento ideológico.

Una vez en la periferia que supone el castillo donde se aloja Gawayn, las escenas de seducción operan como terreno fértil para la parodia. En tres encuentros sucesivos, la señora Bertilak intenta seducir a su huésped, a quien se lo considera, según ella misma señala, epítome de la ideología amorosa cortesana:

"I woled wyr at yow, wyye,» that worthy rher sayde, "And yow wrarhed not rherwyrh, what were the skylle

That so yong and so yepe as ye at this cyme, So conayse, so knyghryly, as ye ar knowen oute

[...] And I haf seten by yourself here sere twyes,

Yet herde I never of your hed helde no wordes

That ever longed to luf, lasse ne more, ${ }^{35}$ (1508 1524).

\footnotetext{
${ }^{32}$ «amistad y fraternidad [...], / pureza y cortesía [...] / y piedad». Armitage, Simon (ed.), op. cit.

33 Caramés Lage, José Luis, «El ritual simbólico y mítico de Sir Gawain and the Green Knight», en Galván Reula, Juan Fernando (ed.), Estudios literarios ingleses. Edad Media, Cátedra, Madrid, 1985, p. 145 (pp. 139-163).

34 Olivares Merino, Eugenio. Del amor, los caballeros y las damas. Hacia una caracterización de la cortaysye en Sir Gawain and the Green Knight, Universidad de Jaén, Jaén, 1999, pp. 234-235.

35 «Me gustaría saber», dijo la noble dama, «y por favor no se ofenda, pero cómo puede ser que un señor tan vivaz y joven -un campeón de la caballería a lo largo del paísque luego de haberme sentado dos veces a su lado. aun así no me ha dirigido ni la más pequeña sílaba relacionada con el amor o algo parecidom»". Armitage, Simon (ed.), op. cit.
} 
Nuevamente, en boca de uno de los habitantes de Gales parece estar la voz que cuestiona el amor cortés como tradición recibida desde los centros hegemónicos. La señora Bertilak expone un desajuste entre lo que Gawayn representa y lo que actúa. Esta brecha produce el efecto paródico junto con la hipérbole que acompaña ambas dimensiones -la exageración con la que la anfitriona describe las cualidades cortesanas de su huésped, por un lado, y la extrema timidez y resistencia del caballero con respecto a sus avances, por otro-. En los tres encuentros sucesivos en los que Gawayn logra detener los intentos de seducción de la señora, este le es leal a su anfitrión, Lord Bertilak, y, con ello, al sistema feudal y a su castidad cristiana. Paradójicamente, esta lealtad implica traicionar las cualidades que lo posicionarían como un héroe en el contexto del romance: su habilidad para seducir a la dama, el trofeo más valioso en el ámbito de los logros cortesanos.

Como resultado de esta paradoja, se pone en funcionamiento una reversión de roles en los que es el caballero el que termina encarnando a la pasiva «damisela en apuros» mientras que su anfitriona debe activamente desafiar e intentar vencer la timidez y castidad de su contrincante. El efecto paródico se refuerza aún más a través del contraste: el sosiego predominante en las tres escenas del dormitorio alterna con la agitación de las escenas de caza, protagonizadas en simultáneo por Lord Bertilak en el espacio exterior. En ambos escenarios alternados se lleva a cabo una batalla: a equipos de caza y gritos salvajes se suceden armas retóricas y diálogos punzantes. Lord Bertilak en el bosque y la señora Bertilak en la habitación se enfrentan a sus respectivas presas, conductas paralelas que dan cuenta del alcance político de lo paródico. En el contexto de la «barbarie» galesa, la mediación del lenguaje y los valores cristianos han vuelto a Gawayn un ser tímido y pasivo. Que el personaje de Gawayn haya sido transformado en presa revela lo peligroso de la violencia salvaje de Gales y lo que esta podría representar para la civilización cristiana anglonormanda. Desde una perspectiva de género, Gawayn no parece responder a los valores patriarcales que lo suponen activo y dominante y termina siendo objeto de las ironías de la anfitriona. En esta línea, los avances de la señora Bertilak se configuran en el contexto de una comunidad cuyas decisiones están tomadas por mujeres: detrás de los anfitriones del castillo se encuentra Morgne Le faye, la hechicera medio hermana de Arthure, que es quien previamente imparte la orden de irrumpir en la corte inglesa y de tentar a Gawayn. La parodia, entonces, expone cómo esta organización pone en jaque al patriarcado anglonormando, amenaza que permite a Inglaterra justificar su ímpetu expansionista de fines del siglo XIV.

No obstante, $S G G K$ no se permite el desarrollo pleno del ejercicio paródico a nivel formal y político. Respecto del primero, Tynianov señala un segundo momento en el despliegue de la 
parodia: «la organización del nuevo material (a este nuevo material pertenece también el viejo material mecanizado)»36. El poema no deviene en una forma artística nueva, sino que se ajusta al verso del romance. Mientras que en España, por mencionar un caso paradigmático, la parodia del romance en El Quijote inaugura la novela moderna, Inglaterra espera hasta el siglo XVIII para dar origen al género ${ }^{37}$ cuando se observa que ni el verso ni el romance pueden hacerse ya de la complejidad del mundo ${ }^{38}$. Si este pasaje es posible dada la consolidación de la burguesía ${ }^{39}$, es entendible que $S G G K$ no proponga una forma nueva porque se alinea con el proyecto expansionista de la aristocracia feudal inglesa. Se produce así, hacia el final del relato, la absorción del elemento subversivo de la parodia a partir de la vuelta a la corte de Gawayn. Las fisuras del relato parecen borrarse cuando Gawayn es recibido como un héroe:

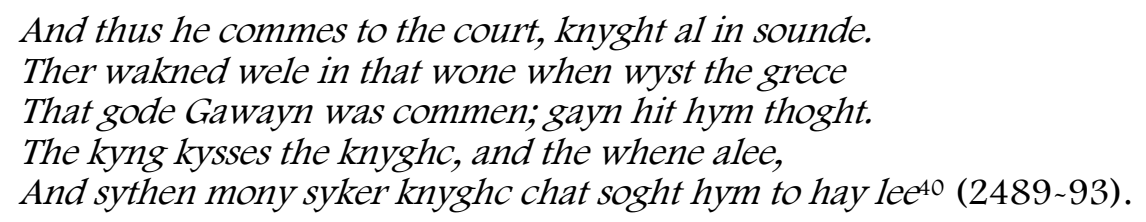

El expansionismo inglés ha conseguido establecer su superioridad en la figura del victorioso Gawayn, lo que aseguraría la conquista de la barbarie galesa. La parodia en sus dimensiones formal y política se anula en el regreso del héroe

Sin embargo, la vuelta del caballero a la corte no obtura completamente el efecto de su contacto con la periferia, lo que sugiere que la fuerza paródica, una vez expuesta, se resiste a desaparecer. El héroe relata su momento de debilidad y el modo en que esto ha sido castigado por el caballero verde. En el último de los encuentros amorosos, la señora Bertilak le regala a Gawayn un cinturón verde que, a su entender, le salvará la vida cuando se enfrente con el caballero verde en la ya próxima decapitación, a condición de ocultárselo a su marido. El caballero inglés, hasta el momento impoluto en su lealtad a su anfitrión, cede a esta tentación y es por eso que el ser sobrenatural, que no es más que el mismo Lord Bertilak, lo castiga produciéndole una leve cicatriz. Así, su triunfo se relativiza dado que su lealtad resquebrajada

\footnotetext{
36 Tynianov, luri, op. cit., p. 169

37 Eagleton, Terry, The English Novel. An Introduction, Blackwell Publishing, Malden, Oxford, Victoria, 2005, p. 7.

38 Ibidem, pp. 2, 12.

39 Ibidem, pp. 7, 11.

40 «Sano y salvo llega a la corte, y cuando los suyos supieron del retorno de su camarada cañones de felicidad hicieron eco en los halls. El rey besó su mano y también la reina, y Gawain fue abrazado por su banda de hermanos». Armitage, Simon (ed.), op. cit.
} 
da cuenta de una falibilidad y no de una condición invencible:

«Lo! lorde,» quoth the leude, and the lace hondeled, "This is the bende of this blame I bere on my nek

This is the lathe and the losse that I laght have

Of couardise and covetyse that I haf caght thare ${ }^{41} . »(2505 \sim 2508)$

La cicatriz es la marca que condensa la desestabilidad formal y política causada por la parodia: el héroe de la ficción y de la nación expansionista no sería tal. Aun así, los caballeros de la corte artúrica se niegan a interpretar la confesión de Gawayn que verbaliza la cicatriz como un fracaso moral y continúan el festejo por el final feliz que implica su retorno. Asimismo, el cinturón de la señora Bertilak, cuyo ocultamiento ha sido para el caballero expresión de su flaqueza en el paródico ejercicio del amor cortés, se vuelve un signo al que el mundo «civilizado» se encuentra con el poder de atribuirle su propio sentido:

The kyng comfortes the knyght, and aile the ourt als,

Laghen loude cherat, and luflyly acorden

That lordes and ladis that longed to the Table,

Uche burne of the brotherhede, a bauderyk schulde have,

$A$ bende abelef hym aboute, of a bryght grene,

And that, for sake of that segge, in swete to were.

For that was acorded the renoun of the Rounde Table,

And he honoured that hit hade, evermore after,

As hit is breved in the best boke of romauncet2 (2513 21).

La vergüenza que inicialmente se desprende de la prenda se resemantiza en un gesto ideológico que sustituye tal humillación por su contrario, un símbolo de heroicidad y pertenencia a la corte de Arthure. En este momento final del romance es la corte en expansión y no Gawayn quien atribuye significados y construye narrativas dominantes. Esta operación política e ideológica del expansionismo inglés borra las fisuras que la parodia ha inscripto en el

\footnotetext{
${ }^{41}$ ««Señor», dijo Gawain, tomando el cinturón, «por eso cargo esta cicatriz

a causa de mi pérdida de fe, me he desfigurado mi físico; debe parecer que me he transformado en un cobarde»». Armitage, Simon (ed.), op. cit.

42 «El rey reconfortó, luego su risa se expandió por el castillo

y en acuerdo amistoso la compañía de la corte permitió que cada lord que perteneciera a su orden, que cada caballero de la hermandad luciera tal cinturón, un brilloso cinturón verde, oblicuo sobre el cuerpo, cruzado, como una banda, por el bien de este hombre. Así, el lazo verde fue adoptado como su insignia, y cada caballero que lo usó fue honrado para siempre, tal como las significativas escrituras de las que el romance nos recuerda». Armitage, Simon (ed.), op. cit.
} 
relato en tanto el reconocimiento de Gawayn de sus propios errores es ignorado y reinterpretado, hasta el punto de que el cinturón adquiere sentidos opuestos. Lo que para el protagonista es signo de su debilidad, para la corte se vuelve señal de fortaleza. Esta le da prioridad a la construcción ideológica afín a sus objetivos por encima de la experiencia de contacto con la otredad protagonizada por Gawayn. El poema se cierra, entonces, anulando las contradicciones del relato expuestas por procedimientos paródicos en pos de los fines políticos y económicos de la expansión anglonormanda.

\section{Consideraciones finales}

Inscripto en el marco de la conformación de Inglaterra como nación a fines del siglo XIV, $S G G K$ presenta el romance como una forma ficcional que se condice con la política expansionista inglesa, anulando el gesto desestabilizador de la parodia que lo atraviesa. Los elementos paródicos estudiados -la subestimación del mundo cortesano artúrico, el desmantelamiento de la figura del héroe, la burla al amor cortés- exponen la caducidad del romance en términos formales y políticos en tanto este se corresponde con el género que canaliza los valores feudales dominantes. No obstante, la parodia se detiene en la objeción y no avanza hacia una postura crítica que postule otra forma ficcional y otro modelo político. Es en este punto donde $S G G K$ se ideologiza al convertirse en vocero del expansionismo aristocrático inglés que insiste en revocar al héroe venido a menos -tal como los valores que ostenta-con un manto conciliador que cubre las marcas del encuentro con un espíritu triunfalista que se pretende vencedor de la alteridad amenazante. 


\section{Bibliografía}

- Armitage, Simon (ed.), Sir Gawain and the Green Knight, W. W. Norton \& Company, Londres y Nueva York, 2007.

- ARNer, Lynn, «The Ends of Enchantment: Colonialism and Sir Gawain and the Green Knight», en Texas Studies in Literature and Language. Vol. 48, No. 2, Verano 2006 (pp. 79 101).

- Caramés Lage, José Luis, «El ritual simbólico y mítico de Sir Gawain and the Green Knight», en Galván Reula, Juan Fernando (ed.), Estudios literarios ingleses. Edad Media, Cátedra, Madrid, 1985 (pp. 139 163).

- Chism, Christine, «Romance», en Scanlon, Larry (ed.), The Cambridge Companion to Medieval English Literature 1100 1500, Cambridge University Press, Nueva York y Cambridge, 2009, (pp. 57 69).

- Davies, Robert R., Domination and Conquest: The Experience of Ireland, Scotland and Wales, 1100 1300, Cambridge University Press, Cambridge, 1990.

- ㄴ The First English Empire. Power and Identity in the British Isles 1093 1343, The Ford Lectures Delivered in the University of Oxford in Hilary Term 1998, Oxford University Press, Oxford, New York, 2000.

- Eagleton, Terry, The English Novel. An Introduction, Blackwell Publishing, Malden, Oxford, Victoria, 2005.

- FunEs, Leonardo, «Medievalismo en el otoño de la Edad Teórica. Consideraciones parciales sobre la operación filológica», en Ciordia, Martín, Cristófalo, Américo, Funes, Leonardo, Vedda, Miguel y Vitagliano, Miguel, Perspectivas actuales de la investigación literaria, Editorial de la Facultad de Filosofía y Letras de la Universidad de Buenos Aires, Buenos Aires, 2011, (pp. 45 72).

- Hahn, Thomas, «Gawain and popular chivalric romance in Britain», en Krueger, Roberta L. (ed.) The Cambridge Companion to Medieval Romance, Cambridge University Press, Cambridge, 2004, (pp.218 234).

- JAMESON, Fredric, «Magical Narratives: Romance as Genre», en New Literary History, Vol. 7, No. 1, Critical Challenges: The Bellagio Symposium, Otoño, 1975, (pp.135 163). 
- LILLEY, Keith, «Imagined Geographies of the «Celtic Fringe» and the Cultural Construction of the 'Other' in Medieval Wales and Ireland», en Harvey, David, Jones, Rhys, McInroy, Neil y Milligan, Christine. Celtic Geographies. Old Culture, New Times, Routledge, Londres y Nueva York, 2005, (pp. 21 36).

- Olivares Merino, Eugenio, Del amor, los caballeros y las damas. Hacia una caracterización de la cortaysye en Sir Gawain and the Green Knight, Universidad de Jaén, Jaén, 1999.

- Tomachevski, Boris, «La construcción de la trama», en Teoría de la literatura, Trad. Suárez, Marcial, Akal, Madrid, 1982, (pp. 179 211).

- Tynianov, Iuri, «Tesis sobre la parodia», en Volek, Emil (ed. y trad.), Antología del formalismo ruso y el grupo de Bajtin. Polémica, historia y teoria literaria. Vol. I Polémica, historia y teoria literaria, Fundamentos, Madrid, 1992, (pp. 169 170). 